\title{
Subcutaneous Sparganosis on Abdomen Mimicking Multiple Lipomas
}

\author{
Seung Ki Ahn, Hwan Jun Choi", Jun Hyuk Kim \\ Department of Plastic and Reconstructive Surgery, College of Medicine, Soonchunhyang University, Cheonan 31151, Korea
}

\begin{abstract}
Human sparganosis is a food-borne zoonosis mainly caused by the plerocercoid belonging to the genus Spirometra. The most common clinical sign of sparganosis is a subcutaneous mass in the trunk including abdominal or chest wall. The mass may be mistaken for a malignant tumor, thereby causing difficulty in terms of diagnosis and treatment. A 66 -year-old woman visited our clinic for the removal of a lipoma-like mass. It was movable, hard, and painless. We identified 2 white mass, measuring $0.2 \times 4 \mathrm{~cm}$ and $0.2 \times 1 \mathrm{~cm}$. Pathologic findings indicated the white mass was a sparganum. She recalled having eaten a raw frog approximately 60 years before. A 35-year-old who lived North Korea was also presented to our clinic with an asymptomatic nodule on her abdomen. Intraoperatively, we found sparganum approximately $24 \mathrm{~cm}$ size. Subcutaneous masses are associated with clinical signs of inflammation or they may mimic a soft tissue neoplasm. While the incidence rate of sparganosis has decreased with economic development and advancements in sanitation, surgeons still encounter patients with sparganosis in the clinical setting. Therefore, a careful history is required in order to diagnose sparganosis.
\end{abstract}

Key words: Sparganosis, subcutaneous tissue, abdominal mass

\section{INTRODUCTION}

Human sparganosis is a food-borne zoonosis mainly caused by the plerocercoid (spargana) belonging to the genus Spirometra [1]. Human sparganosis has been reported in many countries but East Asia is the most common place [2]. The infection is more frequently observed in Korea than in other countries. In humans, the infection is usually due to oral ingestion of larva-containing water or direct ingestion of raw amphibians or reptiles [3]. The most common clinical sign of sparganosis is a subcutaneous mass of body including abdominal or chest wall, scrotum, lower extremity [4]. The spargana are released into the intestine, penetrate the intestinal wall and migrate to subcutaneous and other tissues. The mass may be mistaken for a malignant tumor, thereby causing difficulty in terms of diagnosis and treatment [4]. The infection rate of parasites in Korea has been decreasing recently, but Korean surgeons still occasionally encounter cases of sparganosis. Given

- Received 6 February 2019, revised 4 August 2019, accepted 16 September 2019.

*Corresponding author (medi619@hanmail.net)

(c) 2019, Korean Society for Parasitology and Tropical Medicine

This is an Open Access article distributed under the terms of the Creative Commons Attribution Non-Commercial License (http://creativecommons.org/licenses/by-nc/4.0) which permits unrestricted non-commercial use, distribution, and reproduction in any medium, provided the original work is properly cited. the rarity of the disease, the principles of diagnosis and treatment, as well as clinical characteristics, are not well understood. Here, we report 2 cases of sparganosis with masses located on the abdomen, which were first suspected as lipomas.

\section{CASE REPORT}

\section{Case 1}

A 66-year-old woman visited our clinic for the removal of a lipoma-like abdominal mass that was movable, hard, and painless. Excision and biopsy were planned. Given the small size of the mass, we suspected a lipoma and did not perform any other preoperative tests. Intraoperatively, small incisions were made to explore the abdomen, but no pathologic lesions were identified (Fig. 1). The subcutaneous fat tissue appeared normal, and we identified 2 white masses, one measuring $0.2 \times 4 \mathrm{~cm}$ and another measuring $0.2 \times 1 \mathrm{~cm}$ (Fig. 2). A parasitic infection was suspected, and a biopsy was performed. Pathology results indicated the white mass was a parasite. We requested further information from the division of infectious diseases to identify the exact species of parasite. An antibody screening test was performed, and the results tested positive for sparganum. Following surgery, our patient has not experienced any postoperative complications. She recalled having 


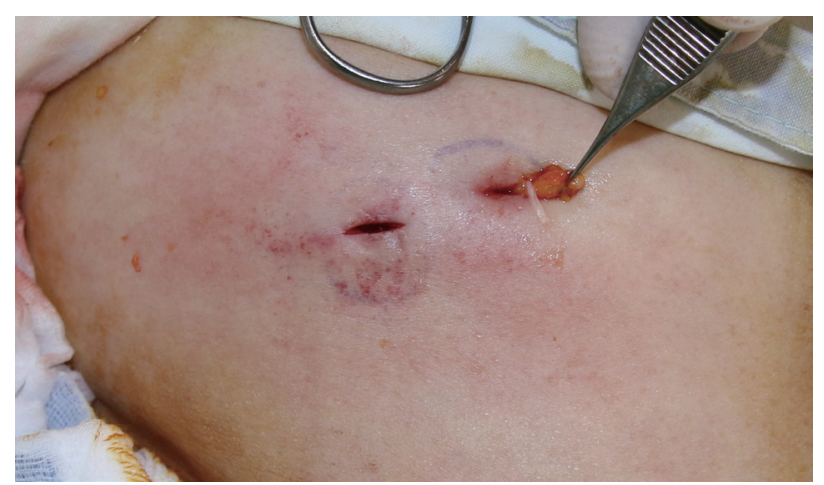

Fig. 1. Intraoperatively, no pathologic findings were observed within the subcutaneous layer; however, a white-lined mass was identified.

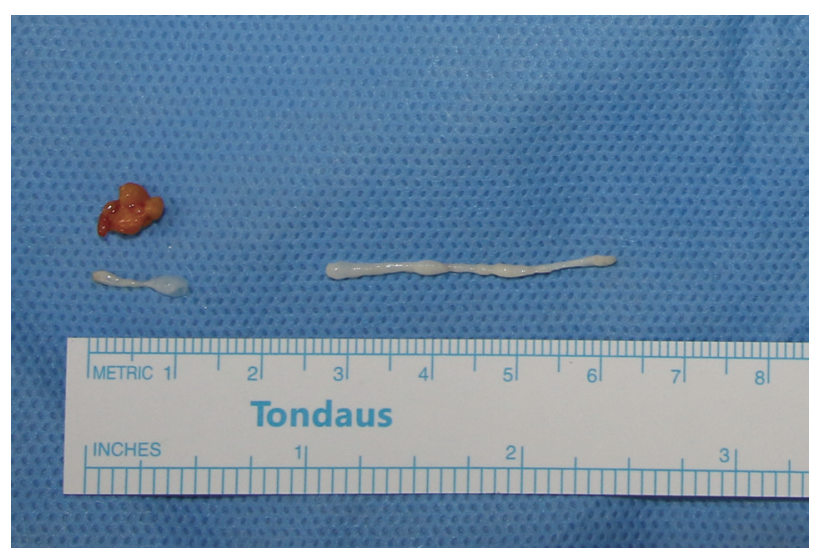

Fig. 2. Normal fat tissue was observed in addition to a $0.2 \times 4 \mathrm{~cm}$ sized white mass and a $0.2 \times 1 \mathrm{~cm}$ sized white mass.

eaten a raw frog approximately 60 years before, but besides that episode, she denied any history of having eaten the raw flesh of snakes or frogs and infected water.

\section{Case 2}

A 35-year-old woman presented to our clinic with an asymptomatic nodule on her abdomen that had been detected a few weeks previously. Physical examination revealed a solitary, moveable subcutaneous nodule, approximately $4 \mathrm{~cm}$ in diameter, and we suspected a lipoma. While performing an excisional biopsy of the lesion and while the incision was being made, a parasite emerged through the incision (Fig. 3). A 24 $\mathrm{cm}$ sized white, flat parasite emerged that was contracting and moving its body, and it was removed (Fig. 4). A histopathological study confirmed the parasite was a sparganum. Following surgery, our patient has had no complications. She denied consuming snake flesh or any kind of relevant high-risk food

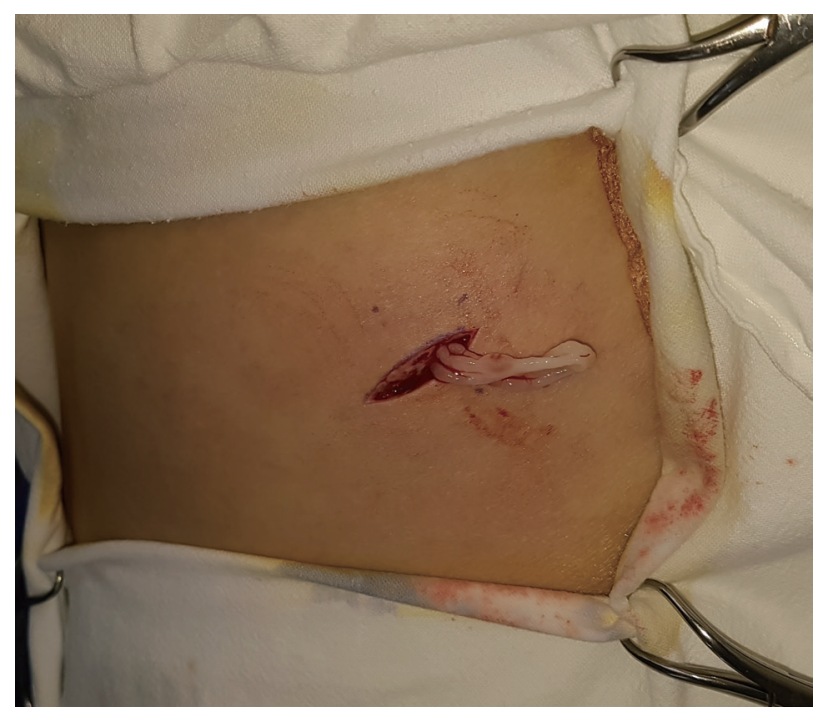

Fig. 3. As an incision was being made, a suspected parasite emerged from the underneath the incision area.

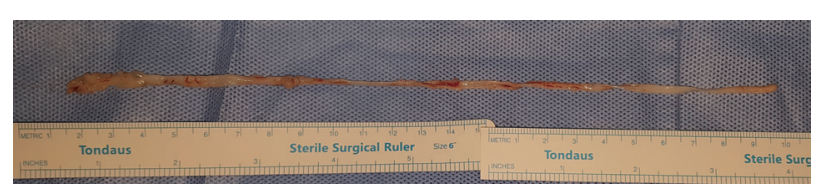

Fig. 4. A $24 \mathrm{~cm}$-sized white, flat sparganum was observed and removed.

including well water, but she previously lived in North Korea where incidents of consuming high-risk food have been recorded as being more common.

An anti-helminthic drug (Praziquantel) was prescribed for prophylaxis, and the postoperative course was uneventful. One year postoperatively, this patient had no symptoms and showed no signs of recurrence of any lesion.

\section{DISCUSSION}

Sparganosis is defined as an infection caused by the larvae of parasitic tapeworms of the Spirometra species. The first case of human sparganosis in Korea was incidentally confirmed by Uemura (1917) in muscle fascia during surgery for a lower extremity amputation in a Korean farmer [5]. The most common location of sparganosis is in subcutaneous tissues [6]. When the spargana is released into intestine, it passes through intestinal wall and moves into subcutaneous tissues, as shown in examinations performed in rats. Humans are an intermediate host for the parasite. Infection occurs via several ways, including drinking water containing infected copepods; eating 
amphibian or rodent meat; and using the flesh of these creatures as a traditional self-treatment according to ritual belief. These risk behaviors have been identified in many reported cases and comprised $41.2 \%$ of reported cases, for example, in Thailand [2].

Park et al. [7] reported that a history of eating raw frog or snake is the most important risk factor for sparganosis, with an odds ratio of 3.1. Sparganosis manifests as a migrating subcutaneous nodule in the abdominal wall, chest wall, lower extremity, or scrotum. It has been reported that ingested spargana can invade various organs, such as the eye, subcutaneous tissues, abdominal wall, brain, spinal cord, lung, or breast. In the case of the genitourinary system, this invasion can include the epididymis, spermatic cord, penis, retroperitoneum, and the ureter. Clinically, patients with sparganosis present with vague or indeterminate symptoms, and distinguishing sparganosis from malignancy remains challenging [4].

The subcutaneous lesion appears as a clear, soft, irregular nodule with a diameter between 0.3 and $5 \mathrm{~cm}$, similar to a fibroma, lipoma, or other neoplasm. With no specific symptom reflecting the presence of larvae in the peritoneum, subcutaneous masses may be the first sign of the disease when the larvae emerge from the peritoneal cavity. Subcutaneous masses are associated with clinical signs of inflammation or they may mimic a soft tissue neoplasm.

Serologic test like ELISA is best tool of diagnosis. Sonographic findings may also be helpful for the preoperative diagnosis of sparganosis. Serpiginous and anechoic tubular tracts with some internal echogenicity are important characteristics. Magnetic resonance imaging characteristics of sparganosis include multiple tubular tracts with low on T1-weighted images and high on T2-weighted images. However, these imaging studies provide limited evidence for making a diagnosis.

Repeated serological tests at appropriate intervals after removal can confirm the removal of the entire sparganum. If the mass is not suitable for surgical therapy, localized chemotherapy with $40 \%$ ethanol procaine or $\alpha$-chymotrypsin can be administered. For patients who have pericardial or pleural effusion without a pleural or pericardial nodule, or who have visceral sparganosis, surgical treatment is not possible [1].

Surgeons should be recognize the possible diagnosis and potential longevity of the spargana. Although the survival duration time of spargana in human skin is not known precisely, there have been case reports of finding a live worm between 8 and 9 years after the development of a subcutaneous mass. We encountered an interesting case of sparganosis that was asymptomatic and latent for 60 years (case 1), and the sparganum was very long ( $24 \mathrm{~cm}$ in size) and was present within the subcutaneous fat layer (case 2). To our knowledge, our report presents the longest latent-occurring sparganosis and reports a patient who was treated for the removal of a very long-living sparganum.

While the incidence rate of sparganosis has decreased with economic development and advancements in sanitation, surgeons still encounter patients with sparganosis in the clinical setting. Therefore, a careful history taking is required in order to diagnose sparganosis.

In conclusion, sparganosis of the subcutaneous tissue is an uncommon disease and is seldom encountered in plastic surgery. Complete surgical removal of sparganosis is the best choice and provides a definite diagnosis. Recurrence depends on the location and the possibility of incomplete removal of the parasite. Once a confirmatory diagnosis is made, a thorough examination of the outer organs, including the abdominal wall and extremities, should be required to detect any concurrent involvement in these locations. Alternatively, a postoperative ELISA test may prevent non-detection of any additional worms.

\section{ACKNOWLEDGMENT}

This study was supported by the Soonchunhyang University Research Fund.

\section{CONFLICT OF INTEREST}

The authors declare no conflict of interest related to this study.

\section{REFERENCES}

1. Liu Q, Li MW, Wang ZD, Zhao GH, Zhu XQ. Human sparganosis, a neglected food borne zoonosis. Lancet Infect Dis 2015; 15 : 1226-1235.

2. Wiwanitkit V. A review of human sparganosis in Thailand. Int J Infect Dis 2005; 9: 312-316.

3. Koo M, Kim JH, Kim JS, Lee JE, Nam SJ, Yang JH. Cases and literature review of breast sparganosis. World J Surg 2011; 35: 573579.

4. Yoon HS, Jeon BJ, Park BY. Multiple sparganosis in an immunosuppressed patient. Arch Plast Surg 2013; 40: 479-481. 
5. Choi SY, Bae JH, Seo BS. Some aspects of human sparganosis in Korea. Korean J Parasitol 1975; 13: 60-77.

6. Kim HY, Kang CH, Kim JH, Lee SH, Park SY, Cho SW. Intramuscular and subcutaneous sparganosis: sonographic findings. J Clin Ultrasound 2008; 36: 570-572.
7. Park HY, Lee SU, Kim SH, Lee PC, Huh S, Yang YS, Kong Y. Epidemiological significance of sero-positive inhabitants against sparganum in Kangwon-do, Korea. Yonsei Med J 2001; 42: 371374. 